\title{
Psychological burden, sexual satisfaction and erectile function in men whose partners experience recurrent pregnancy loss in China: a cross-sectional study
}

Yi-xiang Zhang ${ }^{1}$, Xue-qi Zhang ${ }^{1}$, Qing-rong Wang ${ }^{1}$, Ye-qing Yuan ${ }^{1}$, Jiang-gen Yang ${ }^{1}$, Xiao-wei Zhang ${ }^{2}$ and Qing Li $\mathrm{i}^{*^{*}}$

\begin{abstract}
Background: The aim of this study was to elucidate recurrent pregnancy loss (RPL)-associated psychosocial effects and sexual functions of Chinese men whose partners experience a history of RPL.

Methods: Questionnaire data from a total of 236 men whose partners experience RPL(RPL group) and another 236 non-RPL male volunteers(control group) were analyzed. The self-administered questionnaires included anxiety and depression measures (SAS \& SDS), the Index of Sexual Satisfaction (ISS) and the International Index of Erectile Function (IIEF-5) for evaluating psychological burden, sexual satisfaction and erectile function, respectively.

Results: The mean age of the RPL group and control group was $29.8 \pm 8.6$ and $28.2 \pm 7.3$, respectively. The incidence of erectile dysfunction was significantly higher in the RPL group than in the control group (19.07\% vs. $7.63 \%, P<0.001)$. Anxiety and depression were also more prevalent in RPL group than in the control group (anxiety: $36.90 \%$ vs. $19.08 \%$, $P<0.001$; depression: $26.30 \%$ vs. $7.63 \%, P<0.001$ ). Furthermore, after adjusting for age in the RPL group, negative relationships were observed between the IIEF- 5 score and anxiety and depression $(P<0.001)$, and a positive correlation was found between the ISS and anxiety and depression $(P<0.001)$. In addition, history of RPL, anxiety and depressive symptoms were significantly associated with a higher risk of ED.
\end{abstract}

Conclusions: Psychological functioning, sexual satisfaction and erectile function are impaired in infertile men with RPL partners. These men should be targeted for psychological consultation.

Keywords: Psychological burden, Sexual satisfaction, Erectile function, Recurrent pregnancy loss

\section{Background}

Recurrent pregnancy loss (RPL), defined as two or more consecutive pregnancy losses before the 20th week of pregnancy, is a frequent obstetric complication [1]. Approximately $5 \%$ of women have experienced RPL, and this proportion is even higher among women over 35 years of age [2]. Pregnancy loss has been described as a traumatic event for couples [3]. One-third of women with RPL are diagnosed as depressed $[4,5]$ and $21 \%$ of them have levels of anxiety equal to or higher than that

\footnotetext{
* Correspondence: liqingpku2013@gmail.com

Urology, Peking University People's Hospital, Beijing 100044, China

Full list of author information is available at the end of the article
}

of a typical psychiatric outpatient [4], which in turn may affect sexual functioning and couples' relationships[6]. Moreover, RPL women experiences higher depression and more impaired sexual function compared with the primigravidae [7].

RPL also impacts men; however, their grieving period is shorter than that of women [8]. In a survey of 40 men, $40-59$ \% reported a deepened awareness of the fragility of life, mourned the loss of their family's hopes and dreams and experienced a strong sense of vulnerability and powerlessness [9], although the levels of distress among men are typically less intense than those of women $[5,10]$. RPL also brings negative changes in selfconcept and self-esteem [6]. Coping with involuntary 
childlessness and the stressors associated with treatment may contribute to the difficulties in a couple's relationship and consequently lead to sexual dissatisfaction [11].

However, information about the impacts of RPL on men's psychological status, sexual satisfaction and erectile function is scarce, and most of the previous studies have analyzed women's perspectives exclusively. Hence, the aims of the present study are: (1) to describe the consequences of RPL on the psychological status, sexual satisfaction and erectile function of men; and (2) to explore factors that may contribute to the development of sexual dissatisfaction and psychological dysfunction.

\section{Methods}

\section{Subjects}

Subjects were recruited at two large, general, publicly owned hospitals: Shenzhen People's Hospital and Peking University People's Hospital. Male patients whose partners experience RPL and an equal number of non-RPL male volunteers were recruited. All men had to meet the following criteria: (1) age $\geq 18$ years, (2) in a stable heterosexual relationship for at least 1 year, and (3) able to read and speak Chinese. Their medical histories were evaluated carefully to rule out mental and/or other major medical diseases (e.g., thyroid dysfunction and diabetes mellitus). Men on medication that could have affected their ejaculatory and erectile function and/or psychological status were excluded (e.g., selective serotonin reuptake inhibitors, tricycle antidepressants and phosphodiesterase type 5 inhibitors). The study was approved by the ethical review board at Shenzhen People's Hospital and Peking University People's Hospital. All subjects provided their written informed consent for participation in the study and for the data to be published in the future, and were guaranteed anonymity during data processing.

\section{Procedure}

Subjects completed a psychological questionnaire and a demographic questionnaire including reproductive history. Forms were handed out upon their first visits to the clinic, prior to the investigation of the causes for RPL. Some subjects completed the questionnaires at the clinic; others took them home and returned them to the clinic by mail or E-mail.

\section{Measures}

Personal data from the questionnaire included demographic variables such as age, marital status, height, weight, education level, monthly salary, and stress. To assess psychological burden of all men, the Chineselanguage versions of two standardized and validated questionnaires for depression and anxiety [12] were administered: the Self-rating Anxiety Scale (SAS) [13] and the Self-rating Depression Scale (SDS) [14]. Each questionnaire contained 20 questions. We multiplied the total score on each questionnaire by 1.25 . According to the Chinese norms [12], SAS $\geq 50$ and SDS $\geq 53$ represent diagnosable anxiety and depression, respectively. In addition, the severity of anxiety was categorized as [12]: mild (score 50 - 59), moderate (score 60 - 69), or severe (score $\geq 70$ ). The category ranges for depression were: mild (53-62), moderate $(63-72)$ or severe $(\geq 72)$.

Sexual satisfaction was assessed with the Index of Sexual Satisfaction (ISS) [15], which evaluates respondents' sexual satisfaction with their "real life" relationship. Measures of sexuality-related aspects for husbands address feelings, attitudes and events occurring in the relationship. The ISS comprises 25 items, all of which are statements describing the quality of the respondent's sex life (e.g., I feel that my partner enjoys our sex life). Respondents use seven-point Likert scales to rate the degree to which they agree with each item, with the end-points designated as "All the time" (Score 7) and "None of the time" (Score 1). The score is determined by summing all the item scores, subtracting the number of completed items, multiplying this figure by 100 , and dividing the number of items completed by 6 . This will produce a range from 0 to 100 with higher scores indicating a greater magnitude or severity of problems. Scores below or equal to 30 indicate an absence of a clinically significant problem. Scores above 30 indicate the likelihood of a clinically significant problem. Scores above 70 nearly always indicate that clients are experiencing severe stress with a clear possibility that some type of violence could be considered or used to deal with problems. In this study, the internal consistency of the Chinese-language ISS was 0.96 .

Finally, on the basis of sexual activity status in the preceding four weeks, all men were required to complete the Chinese version [16] of the International Index of Erectile Function (IIEF-5) questionnaire [17]. Erectile dysfunction (ED) was divided into four severity grades [17]: no ED (IIEF-5score, 22 - 25), mild (12 - 21), moderate $(8-11)$, or severe $(5-7)$.

\section{Statistical analyses}

All statistical analyses were performed using SPSS software (SPSS Inc., Chicago, IL, USA) version 13.0. Descriptive statistics were used to summarize the demographic characteristics of the RPL and control groups. Numerical data, normally distributed after verified by the KolmogorovSmirnov test and expressed as mean \pm SD, were compared by unpaired two-sided Student's t tests. The Chi-square test was used to compare categorical data. Correlations between the outcomes of the measures for sexual dysfunction and psychological burden in the RPL group and the control group were assessed using partial correlations. Logistic 
regression was applied for multivariate analysis. Odds ratios $(\mathrm{OR})$ and $95 \%$ confidence intervals $(\mathrm{CI})$ were calculated to examine association strength. Because the ages of RPL men ranged from 20 to 49 years, and sexual dysfunctions are known to be age-dependent phenomena [18], all data were adjusted for age when the correlations between the sexual and psychological problems were assessed. For all of the tests, $P<0.05$ was deemed statistically significant.

\section{Results}

\section{Descriptive results}

From January 2013 to December 2014, 260 men whose partners experience RPL were received the set of study questionnaires at their first clinic visit. A total of 236 men (response rate, $90.8 \%$ ) completed questionnaires. Another 236 male volunteers who had at least one child were enrolled from our health examination center as a control group. Men in RPL group discontinued the study for the following reasons: withdrawal of consent $(N=12)$, loss to follow-up $(N=8)$, incomplete information $(N=2)$, and other reasons $(N=2)$. The background characteristics of the participants are summarized in Table 1.

When compared with men from the control group, men in RPL group reported significantly higher rates of sexual dysfunction and psychological burden, including ED, anxiety, and depression symptoms $(P<0.001$ for all) (Table 2).

The mean ISS scores for the RPL group were higher than those for the control group $(P=0.021)$, indicating that the men from the RPL group were having poorer sexual contact experiences. In this group, 19 (8.05\%) men had scores over 70, which indicated they may have been having significant stress in their daily life.

When erectile function was assessed, the mean IEFF-5 scores for the RPL were lower than the control groups $(P=0.010)$. In the RPL group, a total of 45 men $(19.07 \%)$ reported overall ED (IIEF-5 score <22), and twenty-nine men reported a mild form (IIEF-5 score 12 - 21) and 16 a moderate form (score 8 - 11), whereas no patients reported a severe form (score $5-7$ ). However, in the control group, 18 men $(7.63 \%)$ were diagnosed with ED. Fifteen (6.36 \%) men reported mild ED and three $(1.27 \%)$ a moderate ED, whereas no men reported a severe ED.

According to the SAS and SDS results, $36.90 \%$ (87/ $236)$ and $26.30 \%(62 / 236)$ men of RPL group were diagnosed with anxiety and depression, respectively. Mean scores of the SAS and SDS for men with RPL partners were presented in Table 2. Among them, $20.33 \%$ (48/ 236) reported mild anxiety, $9.32 \%(22 / 236)$ moderate anxiety, and $7.20 \%(17 / 236)$ severe anxiety. For the SDS, $10.60 \%(25 / 236)$ reported mild depression and $15.68 \%$ (37/236) moderate depression, whereas no patients
Table 1 Demographics information of men in RPL group and control group

\begin{tabular}{|c|c|c|c|}
\hline Characteristics & $\begin{array}{l}\text { RPL group } \\
(N=236)\end{array}$ & $\begin{array}{l}\text { Control group } \\
(N=236)\end{array}$ & $P$ value \\
\hline Age, years & & & 0.297 \\
\hline $20-29$ & $137(58.1 \%)$ & $142(60.2 \%)$ & \\
\hline $30-39$ & $63(26.7 \%)$ & $61(25.8 \%)$ & \\
\hline $40-49$ & $36(15.3 \%)$ & $33(14.0 \%)$ & \\
\hline BMl, kg/m2 & $25.32 \pm 5.27$ & $25.51 \pm 5.01$ & 0.802 \\
\hline Cigarette smoking & & & 0.424 \\
\hline Yes & $94(39.8 \%)$ & $102(43.2 \%)$ & \\
\hline No & $142(60.2 \%)$ & $134(56.8 \%)$ & \\
\hline \multicolumn{4}{|l|}{ Alcohol consumption } \\
\hline Yes & $65(27.5 \%)$ & $72(30.5 \%)$ & \\
\hline No & $171(72.5 \%)$ & $164(69.5 \%)$ & \\
\hline Educational status & & & 0.794 \\
\hline Primary school & $15(6.4 \%)$ & $12(5.0 \%)$ & \\
\hline Middle school & $39(16.5 \%)$ & $42(17.8 \%)$ & \\
\hline High school & 78 (33.1 \%) & $83(35.2 \%)$ & \\
\hline University & $104(44.0 \%)$ & $99(42.0 \%)$ & \\
\hline Monthly income (RMB) & & & 0.507 \\
\hline$<3000$ & $51(21.6 \%)$ & $59(25.0 \%)$ & \\
\hline $3000-5000$ & $79(33.5 \%)$ & $81(34.3 \%)$ & \\
\hline$>5000$ & $106(44.9 \%)$ & 96 (40.7 \%) & \\
\hline Length of marriage (year) & $4.43 \pm 3.77(1-19)$ & $4.81 \pm 3.92(1-21)$ & 0.213 \\
\hline $\begin{array}{l}\text { Number of past } \\
\text { pregnancy losses }\end{array}$ & $2.90 \pm 0.97(2-6)$ & $\mathrm{N} / \mathrm{A}$ & \\
\hline $\begin{array}{l}\text { Interval after the latest } \\
\text { loss }\end{array}$ & & N/A & \\
\hline$<1$ month & $46(19.49 \%)$ & & \\
\hline 1-3 months & $122(51.69 \%)$ & & \\
\hline 4-6 months & $57(24.15 \%)$ & & \\
\hline$>6$ months & $11(4.66 \%)$ & & \\
\hline
\end{tabular}

Data were expressed as mean \pm standard deviation or number (percentage) or range, when appropriate

Data were assessed with one-way ANOVA or Chi-square test, when appropriate

$B M I$ body mass index, 1 US dollar $=6.5 \mathrm{RMB}$

reported severe depression. Additionally, the SAS and SDS scores were not reliably associated with the number of past pregnancy losses.

In contrast, the mean SAS and SDS scores reported by men in the control group were lower than those in the RPL group $(P<0.001)$. The incidences of anxiety and depression in the control group were $19.08 \%(49 / 236)$ and $7.63 \%(18 / 236)$, respectively, which were lower than those in the RPL group $(P<0.001$ for all).

In addition, there were several associations among the sexual function and psychological distress variables (Table 3). After adjusting for age, negative relationships 
Table 2 IIEF-5, SAS, and SDS scores of men in RPL group and control group

\begin{tabular}{|c|c|c|c|}
\hline Characteristics & RPL group & Control group & $P$ value \\
\hline ISS & $58.01 \pm 15.11$ & $39.32 \pm 12.27$ & 0.021 \\
\hline IIEF-5, scores & & & $<0.001$ \\
\hline $5-7$ & $0(0 \%)$ & $0(0 \%)$ & \\
\hline $8-11$ & 16 (6.78 \%) & $3(1.27 \%)$ & \\
\hline $12-21$ & 29 (12.29 \%) & 15 (6.36 \%) & \\
\hline $22-25$ & 191 (80.93 \%) & 218 (92.37 \%) & \\
\hline Mean \pm SD & $21.46 \pm 5.96$ & $24.61 \pm 4.71$ & 0.010 \\
\hline SAS, scores & & & $<0.001$ \\
\hline$<50$ & 149 (63.14 \%) & $79.24(21.6 \%)$ & \\
\hline $50-59$ & 48 (20.33 \%) & 25 (10.59\%) & \\
\hline $60-69$ & 22 (9.32 \%) & 16 (6.78 \%) & \\
\hline$\geq 70$ & 17 (7.20 \%) & 8 (3.39 \%) & \\
\hline Mean $\pm S D$ & $39.11 \pm 15.73$ & $24.23 \pm 16.37$ & $<0.001$ \\
\hline SDS, scores & & & $<0.001$ \\
\hline$<53$ & $174(73.73 \%)$ & 218 (92.37 \%) & \\
\hline $53-62$ & 25 (10.59 \%) & 13 (5.51\%) & \\
\hline $63-72$ & 37 (15.68 \%) & $5(2.12 \%)$ & \\
\hline$\geq 72$ & $0(0 \%)$ & $0(0 \%)$ & \\
\hline Mean \pm SD & $26.32 \pm 17.66$ & $12.79 \pm 8.30$ & $<0.001$ \\
\hline
\end{tabular}

Data were expressed as mean \pm standard deviation or number (percentage), when appropriate

Difference between RPL and control groups assessed by Chi-square test or ttest, as appropriate

ISS index of sexual satisfaction, IIEF-5 international index of erectile function, SAS self-rating anxiety scale, SDS self-rating depression scale

were observed between the IIEF-5 score and both SAS and SDS scores; positive relationships were observed between the ISS score and both SAS and SDS scores. Furthermore, plausible predictors for ED (IIEF- $<22$ ) were examined using a multivariate logistic regression model. We found that age, whose partners experience a history of RPL, anxiety and depressive symptoms were significantly associated with a higher risk of ED (Table 4).

\section{Discussion}

This study focused on the consequences of RPL on psychological status, sexual satisfaction, and erectile function, and their possible interrelations among men in China. Results from our study showed that the incidences of ED and psychological problems (anxiety and

Table 3 Associations between the outcomes of the measures for sexual function and psychological distress

\begin{tabular}{llllll}
\hline Characteristics & IIEF-5 & & & ISS \\
\cline { 2 - 3 } & Adjusted $r$ & $P$ & & Adjusted $r$ & $P$ \\
\hline SAS & -0.40 & $<0.001$ & & 0.49 & $<0.001$ \\
SDS & -0.52 & $<0.001$ & & 0.50 & $<0.001$ \\
\hline
\end{tabular}

Table 4 Multivariate logistic regression analysis predicting factors associated with ED (IIEF-5<22)

\begin{tabular}{llll}
\hline Covariates & OR & $95 \% \mathrm{Cl}$ & $P$ value \\
\hline Age & 1.10 & $1.03-1.24$ & $<0.001$ \\
Cigarette smoking & 1.20 & $0.93-1.58$ & 0.142 \\
Alcohol consumption & 0.96 & $0.90-1.06$ & 0.258 \\
RPL history & 1.47 & $1.18-2.29$ & $<0.001$ \\
Anxiety & 1.86 & $1.52-2.78$ & $<0.001$ \\
Depression & 1.58 & $1.28-2.64$ & $<0.001$ \\
\hline
\end{tabular}

depression) in men whose partners experience RPL were higher than those of men in a control group, and a significant association was found between sexual and psychological problems in men with RPL partners.

Our study found that men with RPL partners reported higher incidences of ED and lower sexual satisfaction than did the control group. These incidences were also higher than those reported in a comparable study [19] on the Chinese general population (ED: $13 \%$ ) with subjects of a similar mean age. Similarly, several studies conducted of infertile male patients have reported very similar outcomes. Men in couples who fail to have children experience higher levels of anxiety and depressive symptoms than do fertile men [20]. Similar results have also been published by Dyer et al. [21] from South Africa, where male partners in infertile couples experienced significantly elevated levels of psychological distress compared with fertile men. In addition, ED is reported by one in six infertile patients, mainly associated with depressive symptoms [22]. These findings confirm the data from other studies [21, 23], which suggest that the incidences of sexual and psychological problems in infertile men are higher than those in fertile men. However, it should be noted that different diagnostic criteria for sexual and psychological disorders exist among the various professional organizations and/or individuals. Moreover, the cultural and religious differences between Chinese and Western patient populations should be taken into account. Hence, the difference in prevalence rates can be explained by the previously mentioned factors, and further research is needed to confirm and extend these results.

Although, mechanisms between sexual and psychological problems have not been well studied. The etiology of ED is a combination of psychosocial and organic factors. Corona et al. [24] found that negative psychological factors (e.g., loss or reduced job satisfaction) might impair self-esteem, pushing men to feel disgraced, weakened, and frightened, all of which are negative feelings that can extend further into sexual life, bringing impairments there as well and causing further frustration. Recently, they developed and validated a New SIEDY Scale 3, which is a rapid case history tool able to detect the psychopathological symptoms 
in patients suffering from ED, allowing the identification of those subjects who should undergo an in-depth psychiatric evaluation [25]. Accordingly, we speculate that with the recurrent loss of pregnancies, infertile men might develop feelings of guilt, anxiety and depression. These negative psychological factors might further affect their sexual functioning. Furthermore, diagnostic procedures and treatment attempts may increase psychological distress and hasten the decline in sexual satisfaction. Sexual dissatisfaction could also be related to a withdrawal from sexual activities and hence to even lower chances of conception. However, the cross-sectional nature of our study could not clarify the causal relationship between sexual dysfunction and psychological burden, so further studies on their mechanisms are needed.

\section{Conclusions}

This study was limited by inclusion of a small number of subjects, being a single facility cross-sectional study, and by the use of only self-report assessments. We do not know whether our results can be extended to individuals seeking treatment at other clinics or to those who do not seek treatment at all. Therefore, further in-depth studies on large epidemiological populations are needed to confirm and extend these results. Despite these limitations, this study is noteworthy because, to the best of our knowledge, it is the first research that has comprehensively assessed psychological burden, sexual satisfaction and erectile dysfunction among men whose partners experienced RPL. These findings collectively suggest the importance of psychological care following RPL. Evaluation and management of psychological and psychosocial adjustment of men, and not just women, should be considered in the treatment of RPL to enable partners to adjust emotionally to past pregnancy losses as well as to future pregnancies.

\section{Authors' contributions}

$Y Y, Y Z$, JY and QL conceived the protocol, analysed and interpreted data. QZ and QW made contributions to acquisition of data. XZ revised the paper critically for important intellectual contents. All authors read and approved the final manuscript.

\section{Competing interests}

No potential conflict of interest relevant to this article could be reported.

\section{Author details}

'Urology, The Second Clinical Medical College of Jinan University, Shenzhen People's Hospital, Shenzhen 518020, China. ${ }^{2}$ Urology, Peking University People's Hospital, Beijing 100044, China.

Received: 3 October 2015 Accepted: 19 May 2016

Published online: 13 June 2016

\section{References}

1. Pfeifer S. Definitions of infertility and recurrent pregnancy loss: a committee opinion. Fertil Steril. 2013;99:63.

2. Branch DW, Gibson M, Silver RM. Clinical practice. Recurrent miscarriage. N Engl J Med. 2010;363:1740-7.
3. Lee C, Slade P. Miscarriage as a traumatic event: a review of the literature and new implications for intervention. J Psychosom Res. 1996;40:235-44.

4. Craig M, Tata P, Regan L. Psychiatric morbidity among patients with recurrent miscarriage. J Psychosom Obstet Gynaecol. 2002;23:157-64.

5. Lok IH, Neugebauer R. Psychological morbidity following miscarriage. Best Pract Res Clin Obstet Gynaecol. 2007;21:229-47.

6. Serrano F, Lima ML. Recurrent miscarriage: psychological and relational consequences for couples. Psychol Psychother. 2006;79:585-94.

7. Francisco Mde FMR, Bortoletti FF, Nakamura MU. Sexuality and depression among pregnant women with recurrent spontaneous abortion. Rev Bras Ginecol Obstet. 2014;36:152-6.

8. Klier CM, Geller PA, Ritsher JB. Affective disorders in the aftermath of miscarriage: a comprehensive review. Arch Womens Ment Health. 2002;5:129-49.

9. Speraw SR. The experience of miscarriage: how couples define quality in health care delivery. J Perinatol. 1994:14:208-15.

10. Neugebauer R, Kline J, Stein Z, Shrout P, Warburton D, Susser M. Association of stressful life events with chromosomally normal spontaneous abortion. Am J Epidemiol. 1996;143:588-96.

11. Muller MJ, Schilling G, Haidl G. Sexual satisfaction in male infertility. Arch Androl. 1999:42:137-43.

12. Mingyuan Zhang.Manual of psychiatric rating scale (in Chinese).Vol. 1. Hunan: Hunan Science and Technology Press. 1993. p.258.

13. Zung WW. A rating instrument for anxiety disorders. Psychosomatics. 1971:12:371-9.

14. Zung WW, Gianturco JA. Personality dimension and the self-rating depression scale. J Clin Psychol. 1971:27:247-8.

15. Hudson W. W IOSS: Index of Sexual Satisfaction. Chicago: Dorsey Press; 1982.

16. Yifan H. Practical Andrology (in Chinese). Beijing: Science Press; 2009.

17. Rosen RC, Cappelleri JC, Smith MD, Lipsky J, Pena BM. Development and evaluation of an abridged, 5-item version of the International Index of Erectile Function (IIEF-5) as a diagnostic tool for erectile dysfunction. Int J Impot Res. 1999;11:319-26.

18. Lewis RW, Fugl-Meyer KS, Corona G, Hayes RD, Laumann EO, Moreira Jr ED, Rellini AH, Segraves T. Definitions/epidemiology/risk factors for sexual dysfunction. J Sex Med. 2010;7:1598-607.

19. McMahon CG, Lee G, Park JK, Adaikan PG. Premature ejaculation and erectile dysfunction prevalence and attitudes in the Asia-Pacific region. J Sex Med. 2012;9:454-65.

20. Drosdzol A, Skrzypulec V. Depression and anxiety among Polish infertile couples-an evaluative prevalence study. J Psychosom Obstet Gynaecol. 2009;30:11-20.

21. Dyer S, Lombard C, Van der Spuy Z. Psychological distress among men suffering from couple infertility in South Africa: a quantitative assessment. Hum Reprod. 2009;24:2821-6.

22. Lotti F, Corona G, Rastrelli G, Forti G, Jannini EA, Maggi M. Clinical correlates of erectile dysfunction and premature ejaculation in men with couple infertility. J Sex Med. 2012;9:2698-707.

23. Shindel AW, Nelson CJ, Naughton CK, Mulhall JP. Premature ejaculation in infertile couples: prevalence and correlates. J Sex Med. 2008;5:485-91.

24. Corona G, Mannucci E, Petrone L, Ricca V, Balercia G, Giommi R, Forti G, Maggi M. Psycho-biological correlates of free-floating anxiety symptoms in male patients with sexual dysfunctions. J Androl. 2006;27:86-93.

25. Corona G, Ricca V, Bandini E, Rastrelli G, Casale H, Jannini EA, Sforza A, Forti G, Mannucci E, Maggi M. SIEDY scale 3, a new instrument to detect psychological component in subjects with erectile dysfunction. J Sex Med. 2012;9:2017-26.

Submit your next manuscript to BioMed Central and we will help you at every step:

- We accept pre-submission inquiries

- Our selector tool helps you to find the most relevant journal

- We provide round the clock customer support

- Convenient online submission

- Thorough peer review

- Inclusion in PubMed and all major indexing services

- Maximum visibility for your research

Submit your manuscript at www.biomedcentral.com/submit 